\title{
El tratamiento con implantes en pacientes edéntulos totales mandibulares mediante rehabilitación fija
}

\author{
VELASCO ORTEGA E* \\ PATO MOURELO J** \\ GARCÍA MÉNDEZ A $* * *$ \\ LORRIO CASTRO JM**** \\ CRUZ VALIÑO JM**
}

\begin{abstract}
Velasco Ortega E, Pato Mourelo J, García Méndez A, Lorrio Castro JM, Cruz Valiño JM. El tratamiento con implantes en pacientes edéntulos totales mandibulares mediante rehabilitación fija. Av Periodon Implantol. 2007; 19, 3: 151-159.
\end{abstract}

\begin{abstract}
RESUMEN
Introducción. El objetivo del presente estudio era mostrar los resultados del tratamiento con rehabilitación fija mediante implantes dentales en pacientes edéntulos totales mandibulares. Métodos. 29 pacientes fueron tratados con 190 implantes Microdent $®$ con superficie con chorreado de arena para la rehabilitación fija mandibular. Los implantes fueron cargados después de un periodo de cicatrización de 3 meses. El seguimiento clínico fue al menos de 12 meses.

Resultados. Los hallazgos clínicos indican una supervivencia y éxito de los implantes del 97,9\%. 4 implantes se perdieron durante el periodo de cicatrización. El 58,6\% de las prótesis realizadas fueron atornilladas y el $41,4 \%$ fueron cementadas. Después de un periodo medio de carga funcional de 41,4 meses, no ha habido complicaciones tardías.
\end{abstract}

Conclusiones. Este estudio indica que el tratamiento con rehabilitación fija mediante implantes dentales en pacientes edéntulos mandibulares constituye una terapéutica con éxito.

\section{PALABRAS CLAVE}

Implantes orales, rehabilitación fija, mandíbula edéntula, prótesis sobre implantes, implantología oral.

Fecha de recepción: Agosto 2007

Aceptado para publicación: Septiembre 2007

\section{INTRODUCCIÓN}

El conocimiento de los fenómenos biológicos de la oseointegración y el desarrollo de la implantología oral ha supuesto la posibilidad de establecer y mantener una unión rígida, clínicamente asintomática, entre el hueso y el implante que pueda soportar estructuras protésicas con un éxito importante a largo plazo ${ }^{1}$.

Desde sus comienzos el tratamiento con implantes dentales oseointegrados fue concebido, fundamentalmente, para la rehabilitación de los pacientes con

* Profesor Titular de Odontología Integrada de Adultos. Facultad de Odontología. Director del Máster de Implantología Oral. Universidad de Sevilla.

** Profesor Colaborador Honorario de Odontología Integrada de Adultos. Facultad de Odontología. Universidad de Sevilla.

*** Profesor Asociado de Odontología Integrada de Adultos. Facultad de Odontología. Universidad de Sevilla.

*** Profesor Responsable de Odontología en Pacientes Especiales. Facultad de Odontología. Universidad Alfonso X El Sabio. Madrid. 
edentulismo total provocando un verdadero impacto positivo tanto entre los pacientes como en la profesión dental al proporcionar una alternativa terapéutica a la prótesis completa convencional, sobre todo en la mandíbula por la incapacidad funcional que presentaba en la mayoría de los pacientes ${ }^{2}$.

La rehabilitación protésica con implantes oseointegrados de la mandíbula edéntula total puede ser realizada mediante una opción removible implantorretenida (sobredentadura) o una opción fija (rehabilitación) que exige, por parte del profesional, una compleja decisión sobre el paciente, relacionada con la valoración de factores orales (ej. grado de reabsorción ósea, estudio oclusal) y extraorales (ej. expectativas del paciente, coste económico). Aunque la rehabilitación mandibular fija implantosoportada representa una alternativa muy semejante a la dentición natural; sin embargo, en el tratamiento implantológico del paciente edéntulo mandibular, ambas opciones prostodóncicas sobre implantes, removible y fija, pueden satisfacer plenamente las demandas del paciente edéntulo (función, estética, autoestima) ${ }^{3}$.

La rehabilitación fija de la mandíbula edéntula puede ser realizada con diversos protocolos clínicos con respecto al número de implantes (4-10 implantes) y el tipo de prótesis (ej. resina o cerámica, cementada o atornillada) ${ }^{4}$. De acuerdo al protocolo original de Branemark, 6 implantes son suficientes para ser insertados entre ambos forámenes mentonianos para una prótesis fija implantosoportada atornillada con extremos libres posteriores ${ }^{5}$. Posteriormente este mismo protocolo se ha realizado con 4 o 5 implantes, aunque el diseño de extremo libre posterior puede, por la distribución de las fuerzas, incrementar el riesgo de fracasos biomecánicos ${ }^{6-1}$.

Cuando este estricto protocolo clínico es seguido, la supervivencia de los implantes es elevada, entre un $90 \%$ y $98 \%$. Sin embargo, hay ocasiones en que no es posible insertar 5-6 implantes en la mandíbula edéntula. En estos casos el tratamiento mediante sobredentaduras sobre 2 implantes puede ser una alternativa. La otra posibilidad es realizar técnicas complejas de cirugía implantológica para aumentar la disponibilidad de hueso (ej. injertos óseos, distracción osteogénica) ${ }^{8}$.

Diversos estudios han sido publicados sobre el tratamiento mediante una rehabilitación fija de la mandíbula con implantes oseointegrados ${ }^{2,6-1,9-10}$. Un estudio retrospectivo sueco presentaba los resultados a 10 años con prótesis fija sobre implantes insertados en 2 fases quirúrgicas (sumergidos) en pacientes edéntulos totales. En 71 pacientes se colocaron 13 prótesis fija sobre 4 implantes y 59 sobre 6 implantes en la mandíbula (dependiendo de la disponibilidad ósea), con unos resultados de éxito del $88,4 \%$ y $93,2 \%$, respectivamente, que indican una mayor tendencia en los fracasos entre los pacientes tratados con 4 implantes ${ }^{9}$.

Un estudio prospectivo italiano valora los resultados a 10 años sobre 1286 implantes insertados en una fase quirúrgica (no sumergidos) en 233 pacientes edéntulos totales con diferentes tipos de prótesis, removible (sobredentaduras) y fija. 40 pacientes fueron rehabilitados con prótesis fija sobre 8 implantes en la mandíbula con un éxito del $96,2 \%$, lo que indica que este protocolo representa una buena opción de tratamiento ${ }^{10}$.

El objetivo del presente estudio era la evaluación de los resultados clínicos del tratamiento con rehabilitación fija mediante implantes oseointegrados en pacientes edéntulos totales mandibulares.

\section{PACIENTES Y MÉTODOS}

El presente estudio fue realizado por profesores de las unidades docentes de Odontología Integrada de Adultos de la Facultad de Odontología y del Postgrado de Implantología Oral de la Universidad de Sevilla. Previamente a la realización del estudio, aquellos pacientes que padecían trastornos sistémicos graves que podían comprometer la oseointegración fueron excluidos del estudio ${ }^{11}$. Los pacientes seleccionados eran adultos, de ambos sexos.

Todos los pacientes del estudio eran edéntulos totales mandibulares. Los pacientes fueron informados de la técnica quirúrgica así como del protocolo prostodóncico, la temporalización, el seguimiento clínico, y la posible existencia de complicaciones y pérdidas de implantes. Todos los pacientes fueron evaluados radiológicamente, con una ortopantomografía (Figura l) y en los casos necesarios con una tomografía axial computarizada (TAC). Todos los pacientes autorizaron el tratamiento implantológico mediante un consentimiento informado.

Los criterios de éxito y supervivencia de los implantes fueron los recomendados por van Steenberghe et $\mathrm{al}^{12}$. En este sentido, la supervivencia fue definida como la proporción de implantes permanentes en su localización original aunque no tengan valor clínico o cause efectos adversos. Los criterios de éxito de los implantes se expresan en la Tabla 1. 


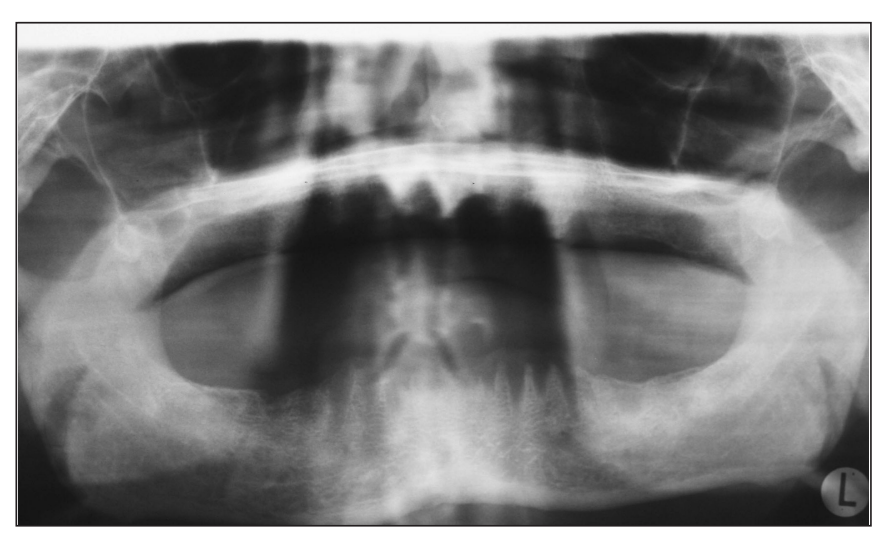

Fig. 1: Ortopantomografía del paciente previa al tratamiento.

\section{Cirugía}

Una hora antes de la cirugía, los pacientes comenzaron un régimen antibiótico preventivo (amoxicilina + clavulánico) durante una semana. En casos de molestias, dolor o inflamación a todos los pacientes se les recomendó ibuprofeno. Todos los pacientes recibieron anestesia local. A todos los pacientes se les recomendó el enjuague diario con clorhexidina durante los primeros 30 días. Los implantes utilizados (de conexión externa con superficie con chorreado de arena) fueron Microdent $₫$ (Microdent, Barcelona, España) (Figuras 2-3). En los casos necesarios se utilizó como bio-

\section{TABLA 1.- LOS CRITERIOS DE ÉXITO EN EL TRATAMIENTO CON IMPLANTES (van Steenberghe et $\mathrm{al}^{12}$ )}

1. El implante no provoca ninguna reacción alérgica, tóxica o infeccciosa de carácter local o sistémico.

2. El implante ofrece soporte para una prótesis funcional.

3. El implante no muestra signos de fractura o incurvación.

4. El implante no muestra ninguna movilidad cuando es explorado manual o electrónicamente.

5. El implante no muestra ningún signo de radiolucidez mediante una radiografía intraoral.

6. La pérdida marginal de hueso (Rx intraoral) y/o la pérdida de inserción (profundidad de sondaje + recesión) no deben perjudicar la función de anclaje del implante o causar molestias para el paciente durante 20 años.

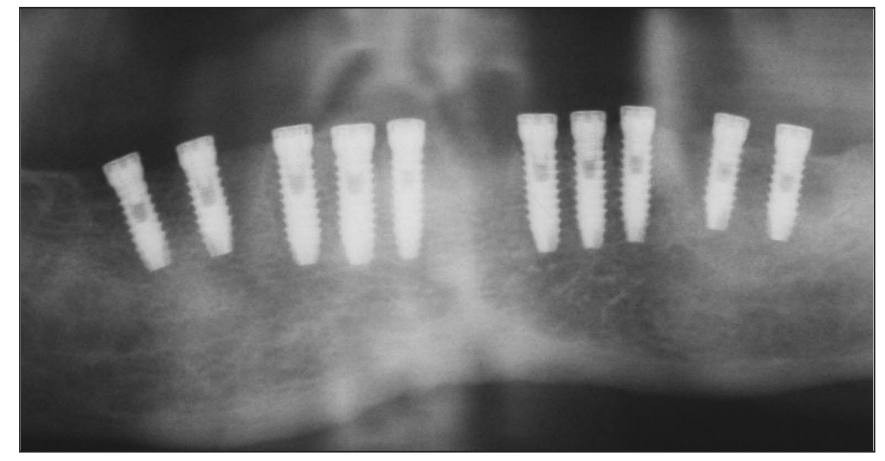

Fig. 2: Aspecto radiológico de los implantes insertados.

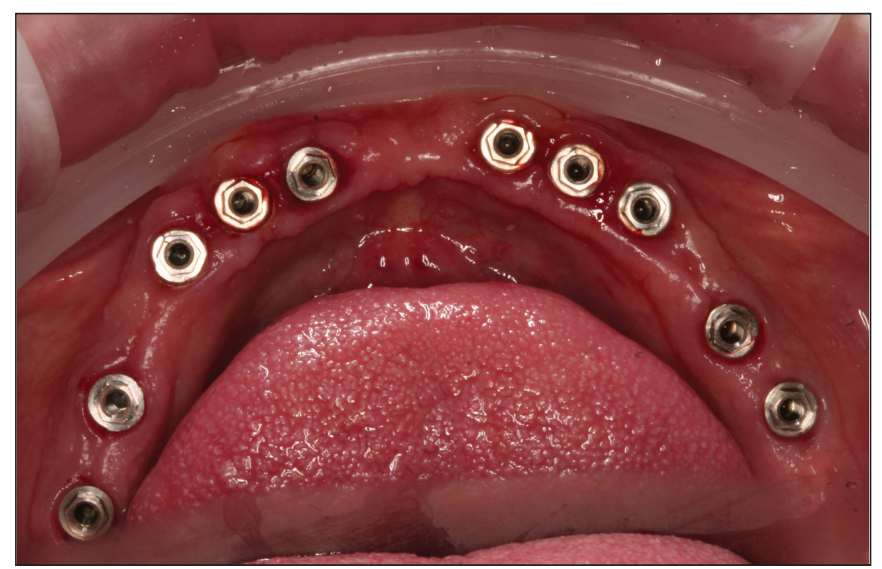

Fig. 3: Aspecto clínico de los implantes después de la fase de cicatrización.

material, el beta-fosfato tricálcico KeraOs $₫$ (Keramat, Coruña, España).

\section{Prostodoncia}

Se realizó una prótesis completa convencional durante el periodo de cicatrización de los implantes. A los 3 meses después de la inserción de los implantes, se realizó la carga funcional de los implantes mediante la colocación de la correspondiente rehabilitación fija en la mandíbula (Figuras 4-6). El tiempo transcurrido de seguimiento clínico desde la carga funcional de los implantes fue al menos de 12 meses.

\section{Análisis estadístico}

Se realizó una estadística descriptiva de los hallazgos clínicos del estudio, con referencia a las variables de- 


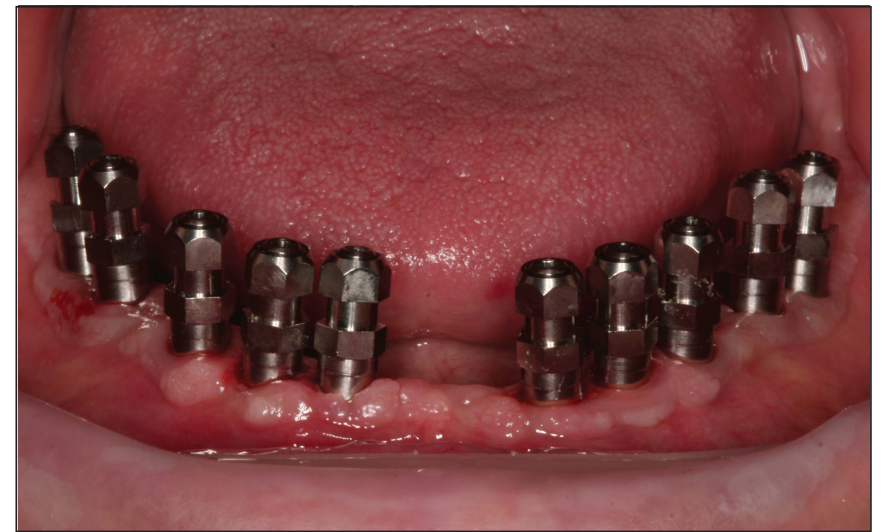

Fig. 4: Fase protésica. Colocación de los postes de impresión.

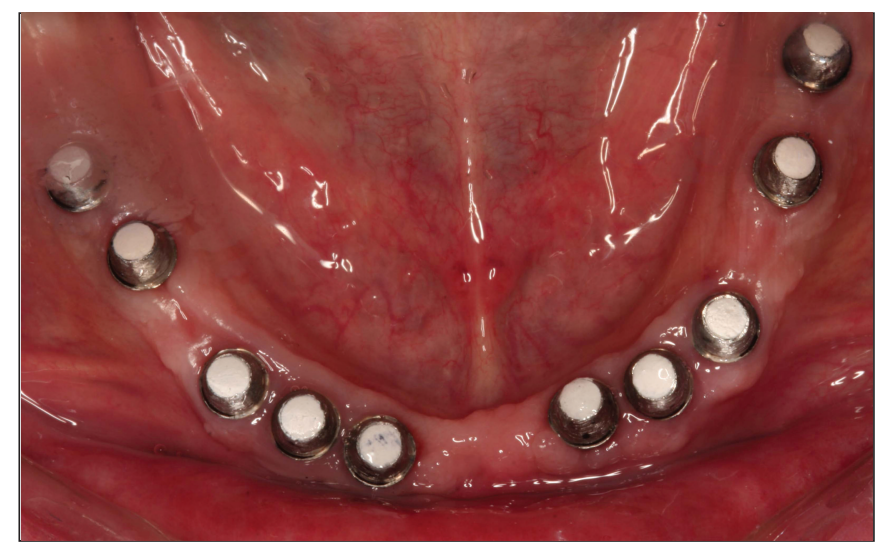

Fig. 5: Fase protésica. Colocación de los pilares sobre los implantes.

mográficas de los pacientes, los implantes y prótesis realizadas.

\section{RESULTADOS}

\section{Pacientes}

29 pacientes edéntulos totales mandibulares (16 hombres y 13 mujeres) fueron tratados con implantes para su rehabilitación prostodóncica fija, con una edad media de 55,8 años (rango: $21-74$ años). 7 pacientes eran fumadores $(24,1 \%) .8$ pacientes habían tenido antecedentes periodontales $(27,5 \%)$.

\section{Implantes}

Se insertaron un total de 190 implantes Microdent ${ }^{\circledR}$ en los correspondientes 29 pacientes, lo que represen-

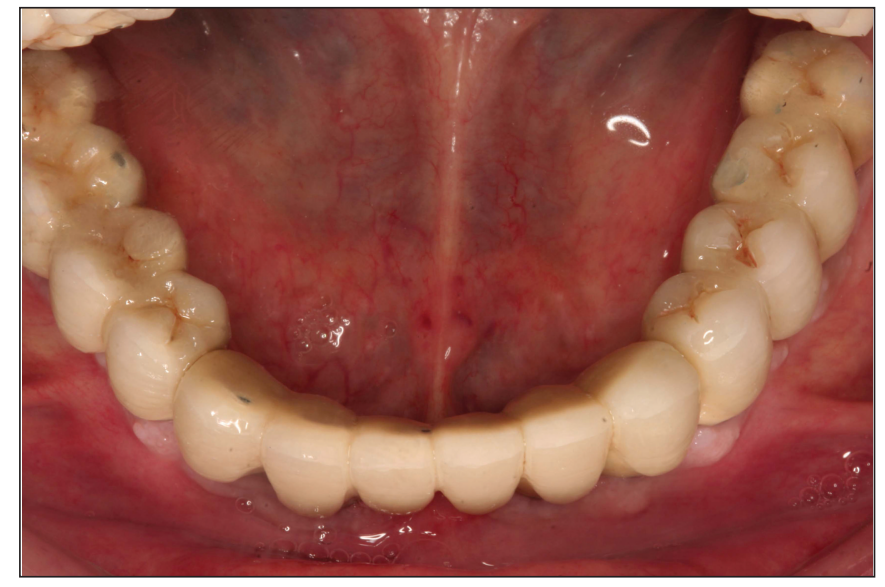

Fig. 6: Fase protésica. Colocación de la rehabilitación oral fija inferior implantosoportada.

ta una media de 6,5 implantes por paciente. Con respecto al diámetro, 179 implantes eran de $4 \mathrm{~mm}(94,2 \%)$, 10 implantes de $5 \mathrm{~mm}(5,2 \%)$ y 1 implante de $3,3 \mathrm{~mm}$ $(0,6 \%)$. Con respecto a la longitud, fueron insertados 10 implantes de $16 \mathrm{~mm}$ (5,3\%), 32 implantes de $14 \mathrm{~mm}$ $(16,8 \%), 115$ implantes de $12 \mathrm{~mm}(60,5 \%)$ y 33 implantes de $10 \mathrm{~mm}(17,4 \%)$.

En 5 implantes (2,6\%) hubo complicaciones, 4 implantes presentaron $(2,1 \%)$ movilidad a la exploración clínica durante la fase de cicatrización libre de carga funcional y fueron extraídos. 1 implante presentó periimplantitis que fue tratada mediante técnica de regeneración ósea sin pérdida posterior del mismo. Ningún implante se ha perdido después de la carga funcional prostodóncica durante el periodo de seguimiento clínico.

\section{Prótesis implantosoportadas}

Los 186 implantes restantes fueron cargados funcionalmente, a los 3 meses. Se realizaron, por tanto, 29 rehabilitaciones fijas mandibulares implantosoportadas, 17 atornilladas $(58,6 \%)$ y 12 cementadas $(41,4 \%)$. El seguimiento clínico medio ha sido de 41,4 meses (rango: 12-98 meses). Solo hubo en 1 paciente una complicación prostodóncica por fractura de un componente de la prótesis.

\section{DISCUSIÓN}

El tratamiento de los pacientes con edentulismo total mandibular mediante la rehabilitación fija con implan- 
tes oseointegrados constituye una alternativa terapéutica odontológica actual que exige una valoración del paciente que integre los aspectos diagnósticos, quirúrgicos, prostodóncicos y de mantenimiento que representan las diversas etapas secuenciales para conseguir el éxito en esta modalidad implantológica oral ${ }^{4}$. En este sentido, cuando se realiza este protocolo integral de los pacientes las expectativas de éxito son muy elevadas como demuestra los resultados del presente estudio con un $97,9 \%$ de supervivencia de los implantes y del $100 \%$ de las prótesis realizadas.

En primer lugar, desde un punto de vista diagnóstico, para la rehabilitación fija con implantes oseointegrados de la mandíbula edéntula es necesaria realizar una historia clínica y exploración completas del paciente que incluya la realización de los modelos de estudio y su montaje en articulador ${ }^{13}$. Así mismo, es imprescindible realizar un diagnóstico por la imagen que proporcione la información necesaria sobre la estructura y morfología óseas de la mandíbula edéntula ${ }^{14}$. En este sentido, todos los pacientes que participaron en el presente estudio fueron diagnosticados mediante una radiografía panorámica y en aquellos casos de reabsorción ósea más severa se utilizó además como diagnóstico por la imagen, la tomografía axial computarizada (TAC).

En el tratamiento con implantes del paciente totalmente edéntulo es recomendable realizar una férula que puede ser utilizada para el diagnóstico radiológico y como guía quirúrgica para una óptima localización de los implantes ${ }^{15}$. En el presente estudio se realizó una férula radiológica-quirúrgica en el $100 \%$ de los casos que permite una mejor distribución de los implantes con unas mejores perspectivas funcionales (carga y distribución de fuerzas) y estéticas con una mejor emergencia de la restauración protésica.

En segundo lugar, desde un punto de vista quirúrgico, la inserción de implantes en la mandíbula edéntula representa una técnica sencilla para el implantólogo con suficiente experiencia clínica. Actualmente, el material quirúrgico implantológico (ej. fresas) ofrece unos patrones excelentes de calidad que unido al diseño autorroscante de los implantes permite su adecuada inserción en la mayoría de las situaciones clínicas ${ }^{10}$.

No obstante, de forma poco frecuente, por diversos factores orales (ej. mala calidad del hueso, fresado incorrecto) y extraorales (ej. consumo de tabaco) pueden aparecer complicaciones agudas o inmediatas que comprometen la estabilidad primaria del implante causando su movilidad y la pérdida del mismo ${ }^{9-10}$. En el presente estudio 4 implantes $(2,1 \%)$ presentaron una movilidad clínica durante la fase de cicatrización y fueron extraídos.

Una intensa reabsorción alveolar mandibular con crestas alveolares estrechas como consecuencia de un edentulismo total durante un largo periodo de tiempo, así como la existencia de estructuras anatómicas como el nervio dentario pueden incrementar la complejidad del tratamiento con implantes oseointegrados y obligar a la realización de técnicas quirúrgicas que hagan posible la inserción de implantes con suficientes garantías de éxito. En este sentido, se han preconizado diversas técnicas como son los injertos óseos, la distracción alveolar y la lateralización del nervio dentario ${ }^{8,16}$.

La cantidad y morfología del hueso mandibular severamente atrófico puede obligar a realizar técnicas quirúrgicas de injertos óseos autólogos así como la utilización conjunta de biomateriales para incrementar el volumen óseo y favorecer el tratamiento con implantes ${ }^{16}$. Dependiendo de las condiciones clínicas los implantes pueden ser insertados en la misma sesión operatoria o después de un periodo de $3-4$ meses ${ }^{8}$.

En el presente estudio no se han realizado técnicas complejas para la inserción de implantes (ej. injertos óseos) ya que la selección de los pacientes no incluía situaciones clínicas de extrema atrofia mandibular, exceptuando la utilización de biomateriales (beta-fosfato tricálcico) como material de relleno en algunos casos de inserción de implantes postextracción o en defectos óseos producidos durante la cirugía.

La longitud de los implantes puede constituir un factor importante en el éxito del tratamiento ya que se ha demostrado una relación directa entre una mayor longitud y unas mejores expectativas de éxito a largo plazo en los pacientes edéntulos completos ${ }^{10}$. En este sentido, el $100 \%$ de los implantes utilizados en el presente estudio tenían una longitud superior a los $10 \mathrm{~mm}$, y el $82,6 \%$ era superior o igual a $12 \mathrm{~mm}$.

Sin embargo, se han realizado estudios de la inserción de implantes cortos en casos de reabsorción severa mandibular que ofrecen la ventaja de una técnica quirúrgica relativamente sencilla y una escasa morbilidad ${ }^{17-18}$. No obstante, la inserción de implantes cortos en casos de reabsorción mandibular extrema ofrece la desventaja fundamental de que se compromete negativamente la ratio entre la longitud del implante y la distancia al plano oclusal provocando una biomecánica desfavorable ${ }^{8,19}$. 
Con respecto al número de implantes, en el presente estudio se insertaron una media 6,5 implantes por paciente, sin embargo este número puede variar según los estudios ${ }^{5-7,10,20}$. En este sentido, además de la estructura ósea mandibular, los factores biomecánicos son importantes. Si el paciente es edéntulo total con completa superior, la rehabilitación de la mandíbula con una prótesis de resina removible atornillada necesita un número menor de implantes (4-6 implantes) insertados entre los forámenes mentonianos ${ }^{5-7,20}$. Al contrario, en casos de pacientes con dentición natural superior o edéntulos parciales maxilares, una rehabilitación fija inferior exige una distribución de los implantes más extensa hacia sectores posteriores y el número de implantes suele ser mayor (8-10 implantes) ${ }^{4,10}$.

El diámetro de los implantes constituye otro factor importante en el tratamiento implantológico porque un mayor diámetro puede incrementar la superficie de contacto entre el hueso y el implante. Es decir, que para la misma longitud los implantes con un diámetro más ancho presentan una mayor área de contacto óseo que los implantes de diámetro reducido ${ }^{21}$. En este sentido, el 99,4\% de los implantes insertados en el presente estudio presentaban un diámetro superior o igual a $4 \mathrm{~mm}$.

En tercer lugar, desde un punto de vista prostodóncico, es importante valorar el espacio interoclusal, sobre todo en los pacientes que son también edéntulos en el maxilar superior ya que una discrepancia notable entre ambos maxilares puede obligar a elegir más conveniente una restauración removible-fija con resina que una rehabilitación fija con porcelana, e incluso en casos muy desfavorables una sobredentadura ${ }^{22}$.

La carga funcional con la prótesis correspondiente representa la culminación en el tratamiento mediante una rehabilitación fija sobre implantes de la mandíbula y debe ser establecida según la valoración integral del paciente y en lo posible con una planificación previa del mismo. En el presente estudio se ha realizado una carga funcional convencional a los 3 meses.

Sin embargo, en otras situaciones clínicas cuando la valoración individual del paciente demuestre unas favorables condiciones quirúrgicas y prostodóncicas, se puede realizar la rehabilitación mandibular implantosoportada con protocolos de carga precoz e incluso inmediata ${ }^{23-25}$. En este sentido, la importancia de los nuevos diseños más anatómicos y nuevas superficies de los implantes con una mayor rugosidad constituye un factor decisivo en la carga precoz e inmediata ya que consiguen, respectivamente, una mayor estabilidad primaria y oseointegración que mejora la unión del implante al hueso mandibular ${ }^{23-25}$.

El tipo de conexión entre la rehabilitación fija y los implantes oseointegrados (cementada o atornillada) en el tratamiento del paciente edéntulo mandibular constituye una decisión individualizada según la experiencia del profesional que debe ser establecida durante el proceso de planificación del tratamiento previamente a la cirugía para evitar situaciones clínicas adversas que comprometan la funcionalidad y estética de la rehabilitación protésica ${ }^{22,26-27}$.

En el presente estudio, de 29 rehabilitaciones fijas mandibulares implantosoportadas, 17 eran atornilladas $(58,6 \%)$ y 12 eran cementadas $(41,4 \%)$. La rehabilitación mandibular fija atornillada sobre implantes presenta la ventaja de su facilidad para poder ser retirada en casos necesarios, sin embargo exige un ajuste pasivo de la estructura protésica con los implantes como consecuencia de un proceso de laboratorio más complejo que siempre es más dificil de conseguir ${ }^{14,26}$. La prótesis implantosoportada cementada ofrece una excelente estética y el ajuste entre la prótesis y los pilares protésicos implantosoportados es más fácil de conseguir y se semeja a las restauraciones convencionales cementadas con dientes naturales tallados ${ }^{14,27}$.

En general, los diversos tipos de prótesis sobre implantes pueden presentar complicaciones aunque no suelen ser muy diferentes en su frecuencia entre cementadas y atornilladas, además los sectores posteriores presentan más complicaciones que los anteriores y deben evitarse los extremos libres ${ }^{28}$.

En cuarto lugar, desde el punto de vista del mantenimiento, la rehabilitación fija con implantes oseointegrados de la mandíbula edéntula exige una permanente revisión del paciente a lo largo de los años. Los controles periódicos son fundamentales para evitar complicaciones y alcanzar tasas de éxito superiores al 95\% 10,20,29-31,. Las revisiones anuales o semestrales (cuando hay complicaciones) permiten mediante la valoración clínica y radiológica controlar la evolución de los tejidos periimplantarios que constituyen la unión permanente de los implantes al hueso mandibular ${ }^{10}$.

La pérdida de hueso marginal representa un hallazgo frecuente en los resultados a largo plazo como indica un estudio sueco a 15 años realizado con 47 pacientes edéntulos rehabilitados con prótesis fija con un éxito 
global del 98,9\% (de 273 implantes insertados con 2 cirugías, 3 implantes fracasaron) y una pérdida marginal ósea de 0,5 mm durante el primer año, y de 0,05 $\mathrm{mm}$ durante los años sucesivos. Esta pérdida era mayor en los implantes anteriores y estaba relacionada con el consumo de tabaco y la pobre higiene oral. Ninguna de las prótesis fracasaron ${ }^{29}$.

Aunque hay estudios a largo plazo que indican un resultado de éxito del $100 \%$ en las prótesis fijas sobre implantes en pacientes edéntulos mandibulares ${ }^{29}$; sin embargo, el mantenimiento prostodóncico siempre es necesario entre los pacientes tratados con rehabilitación fija mandibular implantosoportada como revela un estudio canadiense con un seguimiento medio de 20 años de 33 pacientes edéntulos totales con 29 rehabilitaciones fijas en mandíbula y 4 en maxilar superior ${ }^{31}$. Durante los primeros 7 años, el éxito de las prótesis implantosoportadas fue del $97,8 \%$. Sin embargo, posteriormente 6 prótesis fijas fueron convertidas en sobredentaduras por pérdidas de implantes (3 prótesis) y por función biomecánica desfavorable (3 prótesis) lo que finalmente supuso un éxito del $84,3 \%{ }^{31}$.

En el presente estudio hubo un caso de periimplantitis en 1 implante que fue tratado con éxito mediante regeneración ósea. Los factores generales como son el consumo de tabaco y los antecedentes de enfermedad periodontal unido a la escasa higiene oral pueden constituir un riesgo más elevado en algunos pacientes tratados con prótesis implantosoportada ${ }^{32}$. En este sentido, una pobre higiene oral de los implantes a largo plazo favorece una mayor pérdida de hueso marginal que puede comprometer el éxito del tratamiento implantológico ${ }^{29,31}$.

\section{CONCLUSIONES}

La terapéutica con implantes oseointegrados del edentulismo total mandibular mediante una rehabilitación fija puede constituir una buena opción odontológica que exige una valoración individualizada de cada paciente englobando los diversos aspectos diagnósticos, quirúrgicos, prostodóncicos y de mantenimiento como fases sucesivas para conseguir el éxito del tratamiento implantológico.

\section{ABSTRACT}

Introduction. The aim of this study was to report the outcome of treatment of completely edentulous mandible patients with fixed prostheses supported with dental implants.

Methods. 29 patients were treated with 190 Microdent $\circledR$ sandblasted surface implants for fixed mandible rehabilitation. Implants were loaded after a healing free-loading period of 3 months. The follow-up were at least 12 months.

Results. Clinical results indicate a survival and success rate of implants of $97,9 \% .4$ implants were lost during the healing period. $58,6 \%$ of patients were restored with screw fixed cemented and $41,4 \%$ with fixed prostheses. After a mean functioning period of 41,4 months, no late complications were reported.

Conclusions. This study indicate that treatment with fixed protheses by dental implants in completely edentulous mandible patients constitute a successful treatment.

\section{KEY WORDS}

Dental implants, fixed prostheses, edentulous mandible, implant-support prostheses, implant dentistry.

\section{BIBLIOGRAFÍA}

1. Albrektsson T, Wennerberg A. The impact of oral implants. Past and future, 1966-2042. J Can Dent Assoc 2005; 71: 327-327d.

2. Branemark PI, Hansson BO, Adell R, Breine U, Lindstrom $\mathrm{J}$, Hallen $\mathrm{O}$ et al. Osseointegrated implants in the treatment of the edentulous jaw. Experience from a 10-year period. Scand J Plast Reconstr Surg 1977; 16 (Suppl): 1-132.

3. Zitzmann NU, Marinello CP. A review of clinical and technical considerations for fixed and removable implant prostheses in the edentulous mandible. Int J Prosthodont 2002; 15: 65-72.

4. Misch CE. Mandibular full-arch implant fixed prosthetic options. En: Misch CE. Dental Implant Prosthetics. San Luís: Elsvier Mosby. 2005. pag: 252-64.

5. Branemark PI. Introduction to osseointegration. En: Branemark PI, Zarb GA, Albrektsson T (eds). TissueIntegrated Prostheses: Osseointegration in Clinical Dentistry. Chicago: Quintessence. 1985. Pag: 11-76.

6. Zarb GA, Schmitt A. The longitudinal clinical effectiveness of osseointegrated dental implants: The 
Toronto study. Part I: Surgical results. J Prosthet Dent 1990; 63: 451-7.

7. Eliasson A, Palmquist S, Svenson B, Sondell K. Five-year results with fixed complete-arch mandibular prostheses supported by 4 implants. Int J Oral Maxillofac Implants 2000; 15: 505-10.

8. Stellingsma C,Vissink A, Meijer HJA, Kuiper C, Raghoebar GM. Implantology and the severely resorbed edentulous mandible. Crit Rev Oral Biol Med 2004; 15: 240-8.

9. Branemark PI, Svensson B, van Steenberghe D. Ten-year survival rates of fixed prostheses on four or six implants and modum Branemark in full edentulism. Clin Oral Impl Res 1995; 6: 227-31.

10. Ferrigno N, Laureti M, Fanali S, Grippaudo G. A longterm follow-up study of non-submerged ITI implants in the treatment of totally edentulous jaws. Part I. Ten-year life table analysis of a prospective multicenter study with 1286 implants. Clin Oral Impl Res 2002; 13:260-73.

11. Buser D, von Arx T, ten Bruggenkate C,Weingart D. Basic surgical principles with ITI implants. Clin Oral Impl Res 2000; 11 (suppl): 59-68.

12. van Steenberghe D, Quirynen, Naert I. Survival and success rates with oral endosseous implants. En: Lang NP, Karring T, Lindhe J (eds.). Proceedings of the 3rd European Workshop on Periodontology. Implant Dentistry. Berlin: Quintessence. 1999. pag: 242-52.

13. Weingart D, ten Bruggenkate CM. Treatment of fully edentulous patients with ITI implants. Clin Oral Impl Res 2000; 11 (suppl.): 69-82.

14. Jabero M, Sarment DP. Advanced surgical guidance technology: a review. Implant Dent 2006; 15: 135-41.

15. Almog DM, Torrado E, Meitner SW. Fabrication of imaging and surgical guides for dental implants. J Prosthet Dent 2001; 85: 504-8.

16. Tolman DE. Reconstructive procedures with endosseous implants in grafted bone. A review of the literature. Int J Oral Maxillofac Implants 1995; 10: 275-94.

17. Geertman ME, Boerrigter EM, van Waas MAJ, va Oort RP. Clinical aspects of a multicenter clinical trial of implant-retained mandibular overdentures in patients with severely resorbed mandibles. J Prosthet Dent 1996; 75: 194-204.
18. Deporter D, Watson P, Pharoah M, Todescan R, Tomlinson G. Ten-year results of a prospective study using poroussurfaced dental implants and a mandibular overdenture. Clin Implant Dent Relat Res 2002; 4: 183-9.

19. Chung DM, Oh TJ, Lee J, Misch CE, Wang HL. Factors affecting late implant bone loss: a retrospective analysis. Int J Oral Maxillofac Implants 2007; 22: 117-26.

20. Arvidson K, Bystedt H, Frykholm A, von Konow L, Lothigius E. Five-year prospective follow-up report of the Astra Tech Dental Implant System in the treatment of edentulous mandibles. Clin Oral Impl Res 1998; 9: 225-34.

21. Misch CE, Wang HL, Misch CM, Sharaway M, Lemons J, Judy KW. Rationale for the application of immediate load in implant dentistry: Part II. Implant Dent 2004: 13: 310-21.

22. Wood MR, Vermilyea SG. A review of selected dental literature on evidence-based treatment planning for dental implants: Report of the Committee on Research in Fixed Prosthodontics of the Academy of Fixed Prosthodontics. J Prosthet Dent 2004; 92: 447-62.

23. Szmukler-Moncler S, Piatelli A, Favero GA, Dubruille JH. Considerations preliminary to the application of early and immediate loading protocols in dental implantology. Clin Oral Impl Res 2000; 11:12-25.

24. Velasco E, Medel R, Linares D, Monsalve L, Velasco C. Los implantes de titanio con superficie grabada con ácidos. Un seguimiento clínico de 2 años. Av Perio Impl Oral 2004; 16: 179-86.

25. Velasco E, García A, Segura JJ, Medel R, López J. La carga inmediata en el tratamiento con implantes del paciente edéntulo completo mandibular. Técnica de Maló. Av Perio Impl Oral 2006; 18: 127-34.

26. Misch CE. Principles for screw-retained prostheses. En: Misch CE. Dental Implant Prosthetics. San Luís: Elsevier Mosby 2005. pag: 452-71.

27. Misch CE. Principles of cement-retained fixed implant prosthodontics: natural teeth and implant abutments. En: Misch CE. Dental Implant Prosthetics. San Luís: Elsevier Mosby 2005. pag: 414-51.

28. Nedir R, Bischof M, Szmukler-Moncler S, Belser U, Samson J. Prosthetic complications with dental implants: from an up-to-8-year experience in private practice. Int J Oral Maxillofac Implants 2006; 21: 919-28. 
29. Lindquist LW, Carlsson GE, Jemt T. A prospective 15 year follow up study of mandibular fixed prostheses supported by osseointegrated implants. Clinical results and marginal bone loss. Clin Oral Impl Res 1996; 7: 329-36.

30. Astrand P, Almfeldt I, Brunell G, Hamp SE, Hellem S, Karlsson U. Non submerged implants in the treatment of the edentulous lower jaw. A 2 year longitudinal study. Clin Oral Impl Res 1996; 7: 337-44.

31. Attard NJ, Zarb GA. Long-term treatment outcomes in edentulous patients with implant-fixed prostheses:The Toronto study. In J Prosthodont 2004; 17: 417-24.
32. Schou S, Holmstrup P, Worthington HV, Esposito M. Outcome of implant therapy in patients with previous tooth loss due to periodontitis. Clin Oral Impl Res 2006; 17 (suppl. 2):104-23.

\section{CORRESPONDENCIA}

Prof. Eugenio Velasco Ortega Facultad de Odontología de Sevilla.

C/ Avicena s/n

41009 Sevilla

Tfno: 954481132

E-mail: evelasco@us.es 